\title{
Charging and Discharging Processes in AIN Dielectric Films Deposited by Plasma Assisted Molecular Beam Epitaxy
}

\author{
M. Koutsoureli ${ }^{1}$, A. Adikimenakis ${ }^{2}$, L. Michalas ${ }^{1}$, E. Papandreou ${ }^{1}$, A. Pantazis $^{2}$, G. \\ Konstantinidis $^{2}$, A. Georgakilas ${ }^{2}$ and G. Papaioannou ${ }^{1}$ \\ ${ }^{1}$ Solid State Physics Section, University of Athens, GR-15784, Athens, Greece \\ ${ }^{2}$ IESL -FORTH, GR-71110, Heraklion, Greece \\ E-mail: mkoutsoureli@phys.uoa.gr
}

\begin{abstract}
In the present work the electrical properties of AlN polycrystalline films deposited at low temperatures by plasma-assisted molecular beam epitaxy (PA-MBE) are investigated. The polarization build-up during constant current injection as well as the depolarization process after the current stress have been investigated through monitoring voltage transients in Metal Insulator - Metal (MIM) capacitors, in temperature range from $300 \mathrm{~K}$ to $400 \mathrm{~K}$. Moreover, current-voltage characteristics obtained at different temperatures revealed that charge collection at low fields in these films occurs through variable range hopping.
\end{abstract}

\section{INTRODUCTION}

Aluminum nitride (AlN) piezoelectric thin film is very popular in RF micro-machined resonators and filters MEMS devices. The advantages arise from its high resistivity and piezoelectric coefficient, which is the largest among nitrides, as well as the possibility of being deposited at temperatures lower than $500^{\circ} \mathrm{C}$ and patterned using conventional photolithographic techniques. AlN generally exhibits smaller piezoelectric and dielectric constants and differs from PZT materials in that it is polar rather than ferroelectric. Recently, AlN has been introduced in MEMS switches [1,2] and reliability tests have proved that under low pullin bias or certain polarity the device degradation may be extremely low. The reliability of such capacitive switches has been investigated in several papers [1-3]. The behavior of these devices could not be explained in terms of the usual treatment of dielectric charging and has been attributed to a polarization due to defects connected to dislocation or other structural or point defects in the polycrystalline AlN film [2]. Different techniques such as sputtering [2,4], metal-organic chemical vapor deposition (MOCVD) [5], pulsed laser deposition (PLD) [6], plasma enhanced chemical vapor deposition (PECVD) [7] and molecular beam epitaxy (MBE) [4] have been used so far in order to investigate and improve the properties of AlN films.

The aim of the present work is to investigate the charging and discharging processes in AlN polycrystalline films deposited by PA-MBE method at low temperatures.

\section{THEORETICAL BACKGROUND}

\subsection{Charging Process}

The basic mechanisms involved in the charging process are the Trap-Assisted-Tunnelling (TAT) and the transient component of the Poole-Frenkel (PF) effect that is responsible for the charge redistribution [8]. The hopping conduction, although present, plays a rather minor role in the presence of high electric fields [9]. The simultaneous action of the two mechanisms leads to a spatial charge distribution that was presented for the case of silicon nitride films, for first time in [10].

Adopting the formulation proposed by $\mathrm{R}$. Ramprasad [11] the time dependent current, assuming that TAT is the only operating mechanism, is given by:

$j_{T A T}(t)=q \int_{x=0}^{\infty} \int_{E=-\infty}^{-q F x} N^{f f}(x, E+q F x) \cdot\left(f_{\infty}-f_{0}\right) \cdot \frac{e^{-t / \tau}}{\tau} \cdot \frac{x}{L} d E \cdot d x$

where the current flows in the $\mathrm{x}$ direction, $\mathrm{L}$ is the sample thickness, $\mathrm{N}_{\mathrm{ff}}$ is the field free trap distribution, $\mathrm{E}$ is the energy, $\mathrm{F}$ is the electric field intensity, $\mathrm{f}_{\infty}$ and $\mathrm{f}_{0}$ are the Fermi functions:

$$
\begin{gathered}
f_{\infty}(E)=\left\{1+\exp \left[\left(E-E_{F}\right) / k T\right]\right\}^{-1} \\
f_{0}(E)=\left\{1+\exp \left[\left(E+q F x-E_{F}\right) / k T\right]\right\}^{-1}
\end{gathered}
$$

with $\tau=\tau_{0} \cdot \mathrm{e}^{2 \mathrm{Kx}}, \mathrm{k}, \mathrm{T}$ and $\mathrm{E}_{\mathrm{F}}$ being the Boltzmann's constant, the temperature and the Fermi energy of the metal electrode respectively. The injected charge is assumed to be redistributed by the transient component of Poole-Frenkel conduction, which is derived assuming that a certain fraction of the 
trapped electrons are lost to $\mathrm{PF}$ emission, thus decreasing the density of injected/trapped charges through the TAT mechanism. Since there is a distribution of trap states, the transient component of Poole-Frenkel current density was defined [8] as:

$$
j_{T P F}(t)=q \mu F \int_{x=0}^{\infty} \int_{E=-\infty}^{-q F x} \exp \left\{-\frac{q}{k T}\left(E-\sqrt{\frac{q F}{\pi \varepsilon_{o p t}}}\right)\right\} \cdot n(x, E, t) \cdot d E \cdot d x
$$

where $\varepsilon_{\text {opt }}$ is the high frequency dielectric constant, $\mathrm{n}$ is the density of trapped electrons at a depth $\mathrm{E}$ from the conduction band and the rest parameters are the same as described earlier. Here it must be pointed out that at any given time the PF process alters the trapped distribution of electrons, which in turn alters the TAT process at subsequent times. This obviously means that the simulation of dielectric charging must take into account these mechanisms simultaneously, as proposed in [8]. Here it must be strongly emphasized that the spatial distribution of trapped charge depends on both the applied electric field and temperature.

\subsection{Discharging Process}

The discharging process in the dielectric film of a MEMS capacitive switch takes place under intrinsic electric field, which is lower than the injecting one and decreases continuously with time [11]. The dominant mechanism during this process for high resistivity materials has been found to be the variable range hopping $[11,9]$, which is strongly affected by temperature.

According to a traditional approach to the analysis of charge carrier kinetics in disordered hopping systems, the carrier jump rate $v$ from a starting site of energy $E_{s}$ to a target site of energy $E_{t}$ over the distance $r$ is [12]:

$$
v=v_{0} \cdot \exp (-u)
$$

where $\mathrm{u}$ is a hopping parameter defined as:

$$
u\left(E_{s}, E_{t}, r\right)=2 \gamma r+ \begin{cases}0, & E_{t}<E_{s}+e F r z \\ \frac{E_{t}-E_{s}-e F r z}{k_{B} T}, & E_{t}>E_{s}+e F r z\end{cases}
$$

Here, $\mathrm{F}$ is the intensity of the electric field, $\mathrm{T}$ is temperature, $v_{0}$ the attempt to jump frequency, $\gamma$ the inverse localization radius, e the elementary charge, $\mathrm{k}_{\mathrm{B}}$ is the Boltzmann's constant and $\mathrm{z}=\cos \theta$ with $\theta$ being the angle between the field and the jump direction. The hopping parameter clearly shows that the jump rate is determined by temperature and by the presence and magnitude of electric field intensity, but only if the latter is strong enough in order to significantly change the energy difference between starting and target sites. Moreover the distribution of trapping states in the band gap plays a key issue role on the application of Eq. 5.

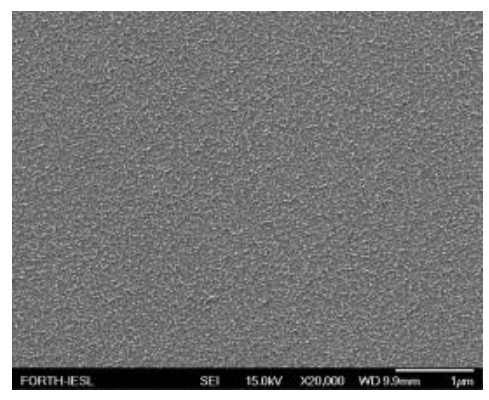

Fig. 1. SEM image of the utilized AIN films.

The temperature dependence of dc conductivity in a disordered system has been predicted by Mott [13] and it has been found to be consistent with the law:

$$
\sigma \propto \exp \left[-\left(\frac{\lambda \cdot \alpha^{3}}{N\left(E_{F}\right) \cdot k_{B} \cdot T}\right)^{1 / 4}\right]
$$

where $N\left(E_{F}\right)$ is the density of states at the Fermi level, $\alpha^{-1}$ is the distance for exponential decay of the wave functions and $\lambda$ is a dimensionless constant, which has been estimated to be approximately 16 [14].

\section{EXPERIMENTAL DETAILS}

Al/AlN samples have been grown on $\mathrm{Si}$ (111) substrates, by PA-MBE. The substrates were chemically cleaned ex-situ and then dipped in aqueous HF solution to remove the surface oxide. In-situ treatment involved heating in UHV up to $900^{\circ} \mathrm{C}$. Then, a thin Al film (400-500 nm) was deposited on the clean $\mathrm{Si}$ surface, at room temperature, followed by an AlN layer with $200 \mathrm{~nm}$ thickness (Fig. 1). The growth process was monitored by RHEED. XRD measurements showed that the AIN layer is single-crystalline (0001), despite the unfavorable growth conditions.

Metal-Insulator-Metal (MIM) capacitors with symmetric Al electrodes were used to assess the electrical properties of the deposited dielectric films. The assessment included current-voltage characteristics for temperatures ranging from $300 \mathrm{~K}$ to $400 \mathrm{~K}$ as well as the measurement of voltage transients during and after current stress of the devices, thus providing information about the polarization build-up during constant current injection and the depolarization of the dielectric film after the stress. All measurements performed in vacuum.

\section{RESULTS AND DISCUSSION}

Current-voltage characteristics obtained at low fields and at different temperatures are presented in Fig. 2a, where the conduction seems to be ohmic. The resulting temperature dependence of the conductivity clearly shows that the charge collection 
at low fields occurs through variable-range hopping mechanism, since the conductivity is consistent to Mott's law (Fig.2b). Ben Hassine et al [15] have also shown that for low electric fields the conduction mechanism in polycrystalline aluminum nitride films is the Ohmic regime and for higher electric fields the ionic conduction seems to be the dominant mechanism, while the Poole - Frenkel transport is identified in the breakdown vicinity.

The voltage transients across the samples have been monitored while stressing the devices at different current levels and after stress, fact that enables us to investigate the build-up of polarization as well as the depolarization procedure. It has been observed [16] that there is a drift of current-voltage characteristics due to current stress in amorphous silicon nitride thin film diodes, due to electron trapping in defect states in the bulk of the dielectric film. The change in voltage $\Delta \mathrm{V}$ that is required in order to maintain a constant injection current, assuming that the trapped charge is uniformly distributed with a concentration $\mathrm{N}_{1}$, is found to be:

$$
\Delta V=\frac{e \cdot N_{1} \cdot d^{2}}{2 \cdot \varepsilon_{0} \cdot \varepsilon_{r}}
$$

where $\mathrm{d}$ is the dielectric film's thickness, e is the elementary charge, $\varepsilon_{0}$ is the vacuum permittivity and $\varepsilon_{\mathrm{r}}$ is the dielectric constant [16] taken as 9.9 [15].

The density of trapped charges in the utilized films has been found to be of the order of $10^{17} \mathrm{~cm}^{-3}$. During current stress the potential build-up, and so
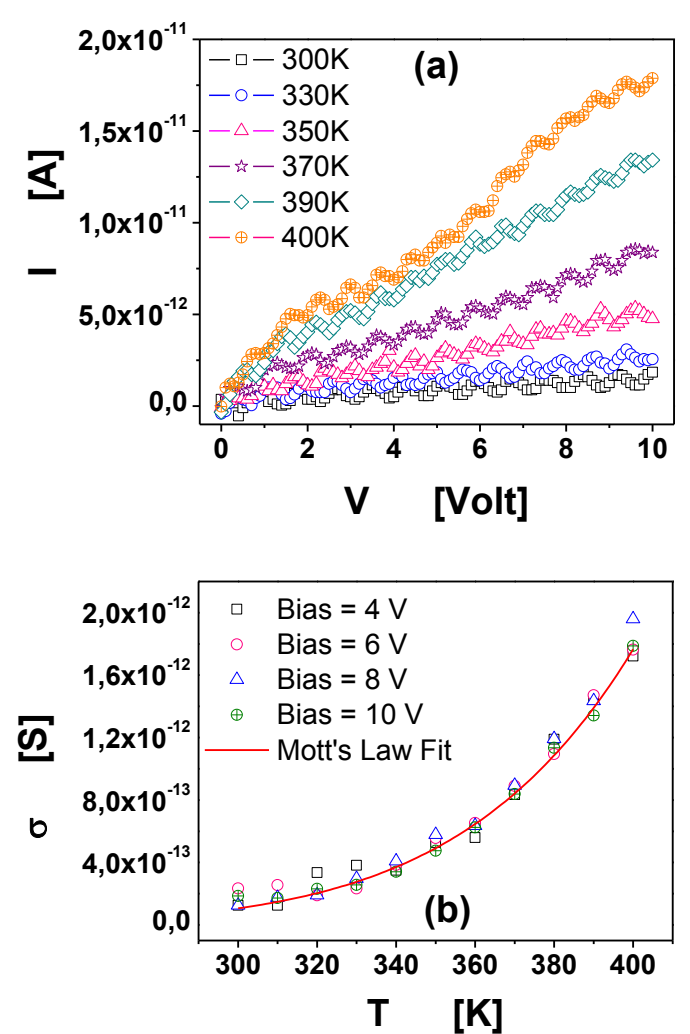

Fig. 2. (a) Current-Voltage characteristics for different temperatures and (b) Temperature dependence of AIN conductivity revealing Mott's law.

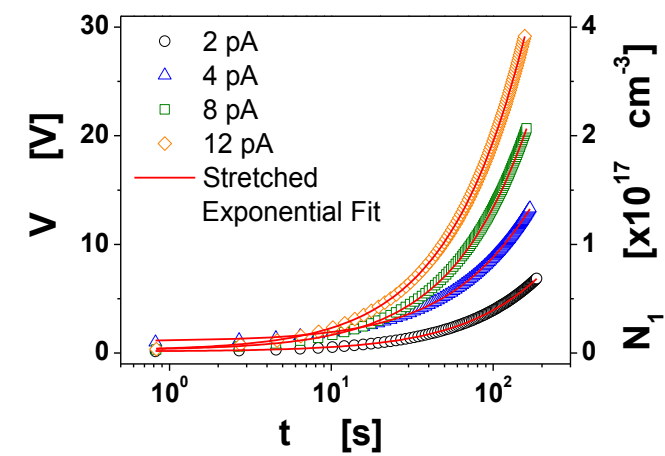

Fig. 3. Build-up of polarization during stress with different current levels and the corresponding density of trapped charges, according to Eq. 7.

the polarization process, obeys a stretched exponential law of the form:

$$
V(t)=V_{\infty}+\left[1-\left(V_{s}-V_{\infty}\right) \cdot \exp \left[-\left(\frac{t}{\tau}\right)^{\beta}\right]\right]
$$

where $V_{\infty}$ refers to instantaneous polarization of the film, $V_{S}$ refers to the static polarization, $\tau$ is the relaxation time and $\beta$ is the stretched factor (Fig. 3). This behavior is in agreement to KohlrauschWilliams-Watts (KWW) polarization's relaxation, found in many materials containing some degree of disorder [17].

The dissipation of charges after current stress has been also monitored by measuring the bias transients after current stress. The depolarization process has been then found to obey a stretched exponential law of the form:

$$
V(t)=V_{0} \cdot \exp \left[-\left(\frac{t}{\tau}\right)^{\beta}\right]+V_{\text {offset }}
$$

where $\mathrm{V}_{0}$ is a fitting parameter representing the initial mean value of the charge distribution (after stress) that gives rise to the monitored transient while $\mathrm{V}_{\text {offset }}$ arises from charges which are collected under very long time constants (inset of Fig. 4). Moreover, the bulk discharge current density has been calculated from the derivation of Eq. 7:

$$
J(t)=\frac{d\left(e \cdot N_{1} \cdot d\right)}{d t}=\frac{2 \cdot \varepsilon_{0} \cdot \varepsilon_{r}}{d} \cdot \frac{d V}{d t}
$$

It has been also found that the bulk discharge current obeys a stretched exponential law of the form:

$$
J_{\text {disch }}(t)=A \cdot\left(\frac{\beta}{\tau}\right) \cdot\left(\frac{t}{\tau}\right)^{\beta-1} \cdot \exp \left[-\left(\frac{t}{\tau}\right)^{\beta}\right]
$$

where $\mathrm{A}$ is a fitting constant. This behavior of depolarization process has been also observed in the dielectric films of MEMS capacitive switches [18] and MIM capacitors [19] with PECVD silicon nitride films. The discharge currents densities obtained for AlN films are found to be in the order of $\mathrm{nA} / \mathrm{cm}^{2}$, which correspond to discharge currents 


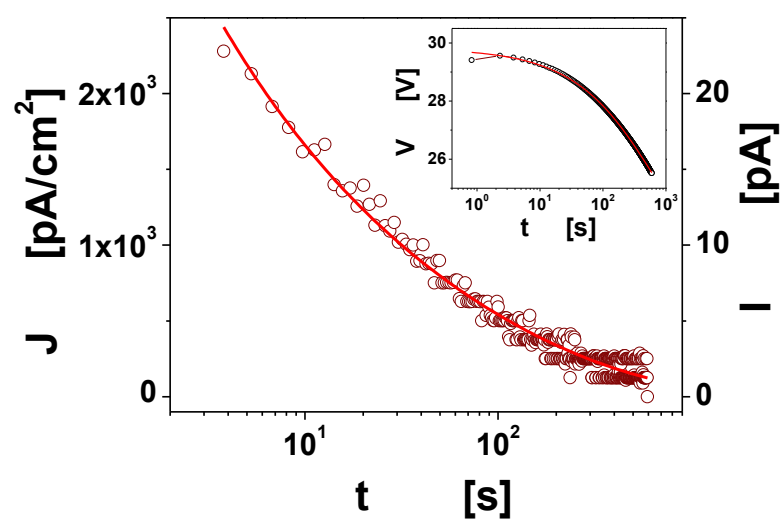

Fig. 4. Bulk discharge current calculated after stressing the device at $12 \mathrm{pA}$. The inset graph presents the decay of potential during the depolarization process. Solid lines represent the fitting of data with Eqs.9 and 11.

of the order of pA [Fig. 4]. These values are three orders of magnitude larger than the corresponding bulk discharge current densities obtained in PECVD SiN films $[9,18,19$,$] .$

\section{CONCLUSIONS}

In the present work the electrical properties of AlN polycrystalline films have been investigated by monitoring current-voltage characteristics for temperatures ranging from $300 \mathrm{~K}$ to $400 \mathrm{~K}$ as well as the voltage transients during and after constant current stress. Charge collection at low fields was found to occur through variable-range hopping mechanism. The build-up polarization behavior during constant current injection in the dielectric film, as well as the depolarization process, are in good agreement to Kohlrausch-Williams-Watts polarization's relaxation, found in many disordered materials. Finally the bulk discharge current density has been found to obey stretched exponential law and it was found to be in the order of $\mathrm{nA} / \mathrm{cm}^{2}$.

\section{Acknowledgements}

The present work has been supported by ENIAC-2010 NANOCOM project and co-financed by the European Union (European Social Fund ESF) and Greek national funds through the Operational Program "Education and Lifelong Learning" of the National Strategic Reference Framework (NSRF) - Research Funding Program: "Heracleitus II Investing in knowledge society through the European Social Fund".

\section{References}

[1] T. Lisec, C. Huth and B. Wagner, "Dielectric material impact on capacitive RF MEMS reliability", in Proceedings of $34^{\text {th }}$ European Microwave Week, 12th GAAS Symp., Amsterdam, The Netherlands, October 2004, pp. 471-474.
[2] E. Papandreou, G. Papaioannou and T. Lisec, "A correlation of capacitive RF-MEMS reliability to AlN dielectric film spontaneous polarization", Int. J. of Microwave and Wireless Technologies, 1(1), pp. 43-47, 2009.

[3] M. Fernandez-Bolanos, D. Tsamados, P. Dainesi and A.M. Ionescu, "Reliability of RF MEMS capacitive switches and distributed MEMS phase shifters using AIN dielectric", in Proceedings of the $22^{\text {nd }}$ IEEE International Conference on Micro Electro Mechanical Systems (MEMS), Sorrento, Italy, January 2009, pp. $638-641$.

[4] M. Placidi, A. Perez-Tomas, J. C. Moreno, E. Frayssinet, F. Semond, A. Constant, P. Godignon, N. Mestres, A. Crespi and J. Millan, "Interfacial properties of AIN and oxidized AIN on Si", Surface Science 604, pp. 63-67, 2010.

[5] C.M. Zetterling, M. Oestling, C.I. Harris, N. Nordell, K. Wongchotigul, M.G. Spencer, "Comparison of $\mathrm{SiO}_{2}$ and $\mathrm{AlN}$ as gate dielectric for SiC MOS structures", Materials Science Forum 264 (2) pp. 877-880, 1998.

[6] Y.F. Lu, Z.M. Ren, T.C. Chong, B.A. Cheong, S.K. Show, J.P. Wang, "Ion assisted pulsed laser deposition of aluminum nitride thin films", Journal of Applied Physics 87 (3) pp. 15401542,2000

[7] G. Sanchez, B. Abdallah, P. Tristant, C. Dublanche-Tixier, M.A. Djouadi, M.P. Besland, P.Y. Jouan, A.B. Alles, "Microstructure and mechanical properties of AIN films obtained by plasma enhanced chemical vapor deposition", Journal of Material Science 44, pp. 6125-6134, 2009.

[8] R. Ramprasad, "Phenomenological theory to model leakage currents in metal-insulator-metal capacitor systems", Physica Status Solidi (b) 239 (1), (2003) 59-70.

[9] M. Koutsoureli, L. Michalas and G. Papaioannou, "The effect of temperature on dielectric charging of capacitive MEMS", 2011 IEEE international reliability physics symposium (IRPS), Monterey CA, USA, April 10-14 2011.

[10] G. Papaioannou, F. Coccetti and R. Plana, "On the modeling of dielectric charging in RF-MEMS capacitive switches", 2010 Topical Meeting on Silicon Monolithic Integrated Circuits in RF Systems (SiRF), N. Orleans LA, USA, January 11-13 2010.

[11] M. Koutsoureli, L. Michalas and G. Papaioannou, "Charge collection mechanism in MEMS capacitive switches", 2012 IEEE international reliability physics symposium (IRPS), Anaheim CA, USA, April 15-19 2012.

[12] M. Pollak, "A percolation treatment of dc hopping conduction", J. of Non Crystalline Solids 11, (1972), 1-24.

[13] N.F. Mott, "Conduction in non-crystalline materials", Philosophical Magazine 19, (1969) 835-852.

[14] V. Ambegaokar, B.I. Halperin and J.S. Langer, "Hopping conductivity in disordered systems", Physical Review B, Vol. 4, No. 8, (1971) 2612-2620.

[15] N. Ben Hassine, D. Mercier, P. Renaux, G. Parat and S. Basrour, "Dielectrical properties of metal-insulator-metal aluminium nitride structures: Measurement and modeling", Journal of Applied Physics 105, (2009) 044111.

[16] J.M. Shannon, S.C. Deane, B. McGarvey and J.N. Sandoe, "Current induced drift mechanism in amorphous $\mathrm{SiN}_{\mathrm{x}}: \mathrm{H}$ thin film diodes ", Appl. Physics Letters 65 (23), (1994) 2978-2980.

[17] H. Kliem, "Kohlrausch relaxations: New aspects about the everlasting story", IEEE Trans. On Dielectrics and Electrical Insulation, Vol. 12 No. 4 (2005) 709-718.

[18] M. Koutsoureli and G. Papaioannou, "Determination of long time discharge current in microelectromechanical system capacitive switches", Appl. Physics Letters 99, (2011) 103503.

[19] U. Zaghloul, M. Koutsoureli, H. Wang, F. Coccetti, G. Papaioannou, P. Pons and R. Plana, "Assessment of dielectric charging in electrostatically driven MEMS devices: A comparison of available characterization techniques" Microelectronics Reliability 50, (2010) 1615-1620. 